\title{
Which characteristics contribute to health-related quality of life in patients with coronary heart disease?
}

\section{Ksenija Tušek Bunc*}

Faculty of Medicine, University of Maribor, Maribor, Slovenia

RECEIVED:

July 28, 2015

ACCEPTED:

September 17, 2015

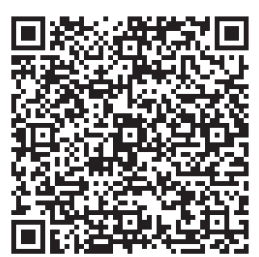

KEYWORDS: quality of life, patients, coronary heart disease.

CITATION: Cardiol Croat. 2015;10(9-10):222-223. | DOI: http://dx.doi.org/10.15836/ccar.2015.222

*ADDRESS FOR CORRESPONDENCE: Ksenija Tušek Bunc, Department of Family Medicine, Faculty of Medicine, University of Maribor, Taborska 8, 2000 Maribor, Slovenia. / E-mail: ksenija.tusek-bunc@guest.arnes.si / Phone: +386-41-699-690

ORCID: Ksenija Tušek Bunc, http://orcid.org/0000-0003-1474-9090

|||||||||||||||||||||||||||||||||||||||||||||||||||||||||||||||||||||||||||||||||||||||||||||||||||||||||||||||||||||||

BACKGROUND: Quality management of patients with coronary heart disease significantly contributes to better health-related quality of life (HRQoL). ${ }^{1,2}$

PATIENTS AND METHODS: The study was cross-sectional and included 423 patients with coronary heart disease from 36 family medicine from all Slovenian regions. The purpose of the study was to identify factors associated with HRQoL at the patient level. A comprehensive questionnaire for patients included, among others, the standardized the EuroQol instrument (EQ-5D) for measuring quality of life. Multivariate regression analysis was performed.

RESULTS: The average age of participants was 68.0 (SD=10.8) years, $64.8 \%$ were male. Multivariate regression analysis of factors impacting the patient, the presence of vascular co-morbidity, and anxietydepressive disorders on HRQoL (as measured by the Visual Analogue Scale), showed (Table 1) significant association between patient age, frequency of family practice visits, heart failure, anxiety-depressive disorders, and the patient chronic illness care evaluation $\left(p<0.001, F=7.816, R^{2}=0.258\right)$. Analysis of the

TABLE 1. Multiple linear regression analysis of prognostic variables with health-related quality of life in patients with coronary artery disease assessed by the Visual Analogue Scale.

\begin{tabular}{lcccccc} 
Patients characteristics & $\mathrm{B}$ & $95 \% \mathrm{Cl}(\mathrm{B})$ & $\mathrm{SE}(\mathrm{B})$ & $\mathrm{B}$ & $\mathrm{t}$ & $\mathrm{p}$ \\
\hline Gender (female) & -0.04 & $(-4.36-4.28)$ & 2.20 & 0.00 & -0.02 & 0.986 \\
\hline Age & $\mathbf{- 0 . 4 2}$ & $\mathbf{( - 0 . 6 0 - - 0 . 2 3 )}$ & $\mathbf{0 . 1 0}$ & $\mathbf{- 0 . 2 3}$ & $\mathbf{- 4 . 3 2}$ & $<\mathbf{0 . 0 0 1}$ \\
\hline Years of education & 2.80 & $(0.00-5.60)$ & 1.43 & 0.10 & 1.96 & 0.050 \\
\hline Marital status & -1.62 & $(-6.31-3.08)$ & 2.39 & -0.04 & -0.68 & 0.499 \\
\hline Being patient in practice $>$ 10 years & 1.85 & $(-2.46-6.16)$ & 2.19 & 0.04 & 0.84 & 0.399 \\
\hline Practice attendance within 12 months & $\mathbf{- 4 . 3 1}$ & $\mathbf{( - 6 . 9 6 - - 1 . 6 5 )}$ & $\mathbf{1 . 3 5}$ & $\mathbf{- 0 . 1 5}$ & $\mathbf{- 3 . 1 9}$ & $\mathbf{0 . 0 0 2}$ \\
\hline Angina pectoris & 1.68 & $(-2.05-5.41)$ & 1.90 & 0.04 & 0.89 & 0.376 \\
\hline Myocardial infarction & 2.68 & $(-1.08-6.44)$ & 1.91 & 0.07 & 1.40 & 0.161 \\
\hline Stent/By pass & -3.62 & $(-7.53-0.28)$ & 1.98 & -0.09 & -1.83 & 0.069 \\
\hline Heart failure & $\mathbf{- 6 . 8 6}$ & $\mathbf{( - 1 0 . 9 1 - - 2 . 8 0 )}$ & $\mathbf{2 . 0 6}$ & $\mathbf{- 0 . 1 7}$ & $\mathbf{- 3 . 3 2}$ & $\mathbf{0 . 0 0 1}$ \\
\hline Stroke & 2.54 & $(-4.89-9.97)$ & 3.78 & 0.03 & 0.67 & 0.502 \\
\hline Peripheral artery disease & -1.85 & $(-5.95-2.25)$ & 2.08 & -0.04 & -0.89 & 0.376 \\
\hline Anxiety / Depression & -10.28 & $(-14.17--6.39)$ & 1.98 & -0.25 & -5.20 & $<0.001$ \\
\hline Medication & -1.17 & $(-12.25-9.92)$ & 5.63 & -0.01 & -0.21 & 0.836 \\
\hline Patient satisfaction (EUROPEP) & $\mathbf{- 0 . 8 5}$ & $\mathbf{( - 4 . 6 1 - 2 . 9 1 )}$ & $\mathbf{1 . 9 1}$ & $\mathbf{- 0 . 0 3}$ & $\mathbf{- 0 . 4 5}$ & $\mathbf{0 . 6 5 6}$ \\
\hline Patient assessment of chronic illness care (PACIC) & 2.47 & $(0.05-4.89)$ & 1.23 & 0.12 & 2.01 & 0.045 \\
\hline
\end{tabular}

$R^{2}=0.258 ; F=7.816 ; p<0.001$

$95 \% \mathrm{Cl}=95 \%$ confidence interval; $\mathrm{SE}=$ standard error 
influence of these factors on health-related quality of life using EQ-5D showed (Table 2) a statistically significant positive association between health self-evaluation and patient education, and a negative association with peripheral artery disease and heart failure ( $\left.\mathrm{p}<0.001, \mathrm{~F}=18.386, \mathrm{R}^{2}=0.450\right)$.

DISCUSSION: HRQOL in patients with coronary artery disease is associated with patient characteristics, with vascular co-morbidity and anxiety-depressive disorders. Of the demographic factors related to HRQoL, older age is associated with a worse HRQoL. Another factor predicting a worse HRQoL is frequency of family practice visits. On the other hand, higher education, better self-assessment of health, and better assessment of chronic illness care are associated with a better HRQoL. Heart failure, peripheral artery disease, and anxiety-depressive disorders stand out as diseases predicting of a worse HRQOL.

TABLE 2. Multiple linear regression analysis prognostic variables with health-related quality of life of patients with coronary artery disease (EuroQol instrument).

\begin{tabular}{lcccccc} 
Patients characteristics & $\mathrm{B}$ & $95 \% \mathrm{Cl}(\mathrm{B})$ & $\mathrm{SE}(\mathrm{B})$ & $\mathrm{B}$ & $\mathrm{t}$ & $\mathrm{P}$ \\
\hline Gender (female) & -0.03 & $(-0.07-0.00)$ & 0.02 & -0.08 & -1.72 & 0.087 \\
\hline Age & 0.00 & $(0.00-0.00)$ & 0.00 & -0.01 & -0.18 & 0.857 \\
\hline Years of education & $\mathbf{0 . 0 3}$ & $\mathbf{( 0 . 0 1 - 0 . 0 6 )}$ & $\mathbf{0 . 0 1}$ & $\mathbf{0 . 1 3}$ & $\mathbf{2 . 9 7}$ & $\mathbf{0 . 0 0 3}$ \\
\hline Marital status & 0.03 & $(-0.01-0.07)$ & 0.02 & 0.06 & 1.37 & 0.172 \\
\hline Being patient in practice $>10$ years & 0.00 & $(-0.04-0.04)$ & 0.02 & 0.00 & -0.03 & 0.973 \\
\hline Practice attendance within 12 months & -0.02 & $(-0.04-0.00)$ & 0.01 & -0.08 & -1.87 & 0.062 \\
\hline Angina pectoris & -0.02 & $(-0.05-0.01)$ & 0.02 & -0.05 & -1.24 & 0.216 \\
\hline Myocardial infarction & -0.03 & $(-0.06-0.00)$ & 0.02 & -0.07 & -1.80 & 0.073 \\
\hline Heart failure & $-\mathbf{0 . 0 5}$ & $\mathbf{( - 0 . 0 8 - - 0 . 0 2 )}$ & $\mathbf{0 . 0 2}$ & $-\mathbf{- 0 . 1 3}$ & $\mathbf{- 2 . 9 2}$ & $\mathbf{0 . 0 0 4}$ \\
\hline Stroke & 0.01 & $(-0.05-0.07)$ & 0.03 & 0.02 & 0.39 & 0.698 \\
\hline PAD & $-\mathbf{0 . 0 6}$ & $\mathbf{( - 0 . 1 0 - - 0 . 0 3 )}$ & $\mathbf{0 . 0 2}$ & $\mathbf{- 0 . 1 5}$ & $\mathbf{- 3 . 6 4}$ & $<\mathbf{0 . 0 0 1}$ \\
\hline Self-percived health & $\mathbf{0 . 1 0}$ & $\mathbf{( 0 . 0 8 - 0 . 1 2 )}$ & $\mathbf{0 . 0 1}$ & $\mathbf{0 . 4 5}$ & $\mathbf{1 0 . 2 4}$ & $<\mathbf{0 . 0 0 1}$ \\
\hline Medication & 0.03 & $(-0.06-0.12)$ & 0.05 & 0.03 & 0.72 & 0.475 \\
\hline Patient satisfaction (EUROPEP) & 0.00 & $(-0.03-0.04)$ & 0.02 & 0.01 & 0.27 & 0.785 \\
\hline Patient assessment of chronic illness care (PACIC) & -0.01 & $(-0.03-0.01)$ & 0.01 & -0.03 & -0.61 & 0.543 \\
\hline
\end{tabular}

$R^{2}=0.450 ; F=18.386 ; p<0.001$

$95 \% \mathrm{Cl}=95 \%$ confidence interval; $\mathrm{SE}=$ standard error 